\title{
Portal Vein Thrombosis and Gut Microbiota: Understanding the Burden
}

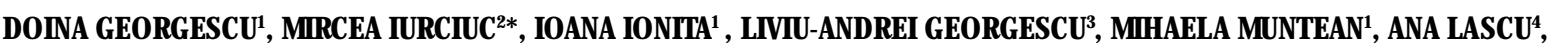 \\ MIHAI IONITA', DANIEL LIGHEZAN ${ }^{1}$ \\ ${ }^{1}$ Victor Babes University of Medicine and Pharmacy, Department of Internal Medicine, 2 Eftimie Murgu Sq., 300041,Timisoara, \\ Romania \\ ${ }^{2}$ Victor Babes University of Medicine and Pharmacy, Department of Cardiology, 2 Eftimie Murgu Sq., 300041,Timisoara, Romania \\ ${ }^{3}$ Victor Babes University of Medicine and Pharmacy, Department of Urology, 2 Eftimie Murgu Sq., 300041,Timisoara, Romania \\ ${ }^{4}$ Victor Babes University of Medicine and Pharmacy, Department of Physiopathology, 2 Eftimie Murgu Sq., 300041,Timisoara, \\ Romania
}

\begin{abstract}
Portal vein thrombosis (PVT) continues to be challenging, due to its versatile features and associated clinical pitfalls. This study aimed to explore whether gutmicrobiota imbalance (GMI) could alter clinical presentation and short term outcome in cirrhotic patients with PVT. We conducted a cross-sectional exploratory study, concerning 50 consecutive cirrhotic patients with PVT recruited during hospitalization. Consecutive to stool microbiological exams, they were assigned into two groups: $25 \mathrm{GMI}$ positive as study group and $25 \mathrm{GMI}$ negative as comparison group. Clinical examination, laboratory work-ups, upper and lower digestive endoscopy, abdominal Duplex ultrasound and CT/MRI were performed Results: Study group displayed significant differences concerning age, interleukin-10 and albumin levels, digestive bleeding, abdominal pain severity and longer hospitalization. In conclusion, dysbiotic patients displayed clinical particularities characterized by older age, higher inflammatory status, hypoalbuminemia, more severe PVT, abdominal pain and digestive bleeding, as well as longer hospitalization duration.
\end{abstract}

Keywords:gut microbiota imbalance, liver cirrhosis, portal vein thrombosis

Portal vein obstruction syndrome (PVOS), most of the times associated to a portal vein thrombosis (PVT), continues to be a challenge, even for the most experienced physicians, due to its diverse etiology and clinical presentation[1]. PVOS is the result of an endoluminal onset and rarely a consequence to an external compression. In the onset of a vascular thrombosis, no matter its location, Virchow postulated the importance of the three factors, called triad: vascular stasis, hypercoagulability and endothelial injury[2].

Stasis in PV territory with decreased velocity of the PV flow and congestion index augmentation is a possibility, frequently seen in patients with end stage liver disease and extended fibrosis (F4) [3]. The risk for PVT seems to increase proportionally to severity of liver fibrosis, $25-40 \%$ of patients with end stage liver diseases, some of them waiting for liver transplantation, being already diagnosed with this condition[4-7]. However, in the onset of PVT, direct tumor invasion by proximity cancer either primary or secondary, metastatic, is often seen. The propensity of PV invasion in patients with hepatocellular carcinoma and liver cirrhosis is well known[8]. PVOS classification has been redefined by several approaches, one of them being Baverno $\mathrm{V}$ consensus on portal hypertension condition, based on PV anatomy, in intra and extra hepatic PV obstruction[9]. Ponziani et al also highlighted the importance of the extension of thrombotic process, classifying PVT accordingly[10]. PVT is an infrequent condition, with the real incidence still unknown, depending on diagnostic approach, between $1 \%$, in consecutive autopsy series, to $1.74 \%$ in CT exam analysis[11,12].The obstruction, either complete or incomplete, due to a local thrombosis, more or less extended is frequently seen, but other factors (local or distant) may also be involved. It is also possible that vascular damage to start when local infections, inflammation or injuries-like trauma are in progress. Apart from classic thrombogenic multiple risk factors, gut microbiota imbalance (GMI), also known as dysbiosis is lately linked to alteration of platelets reactivity and development of thrombosis. Microbes may act by metabolism independent or dependent pathways. The former is related to bacterial translocation and endotoxemia, whereas the latter involves the ability of choline transformation in trimethylamine (TMA) that is further oxidized to trimethylamine $\mathrm{N}$-oxide (TMAO) by hepatic flavin monooxygenases. High TMAO levels were detected in patients with major cardio-vascular risk secondary to thrombotic events[13].

\section{Experimental part}

We have conducted a cross-sectional, exploratory study aiming to determine whether GMI could influence clinical presentation and short term outcome in cirrhotic patients with PVT. A consecutive sample recruitment of 50 cirrhotic patients with PVT was performed during hospitalization in an Internal Medicine Department, University Hospital. Consecutive to stool microbiologic examination with assessment of GMI, participants of the study were assigned into two groups: 25 patients GMI positive (study group) and 25 patients GMI negative (comparison group), as matched pairs. Patients under antibiotic or probiotic treatment at the moment of PVT confirmation were not included in this study. A thorough clinical examination and lab work- ups were performed: complete blood count (CBC), erythrocyte sedimentation rate (ESR), interleukin 10 (IL-10), international normalized ratio ( INR), activated partial thromboplastin time (APTT), D-dimers, transaminases: ASAT, ALAT, total bilirubin, protein ELFO, 
creatinin fast blood sugar, as well as HBs Ag, Delta Ag, anti HCV antibodies, plasma iron and copper, alpha 1 antitripsine, antinuclear antibodies (ANA), antimitochondrial antibodies (AMA), using standard accredited laboratory methods. An extended study of clotting factors, ,as thrombophilia panel : protein C,S, AT III, mutation gene factor $V$ Leyden G1691A, mutation gene prothrombin G20210A, antiphospholipidic antibodies, anticoagulant lupus was ordered in selected patients. Upper and lower digestive endoscopies (Stolz video endoscopes), abdominal ultrasound examination (General Electric Logiq $\mathrm{E}$, high resolution ultrasound equipment, 3,5-5 MHz, convex array probes), as well as abdominal CT and MRI were performed. Each patient experienced the same protocol for ultrasound examination, respectively a Duplex abdominal procedure consisting in a complete plain ultrasound exam in conjunction with Doppler (color, power, pulsate), performed in the morning, after 8 hours of fasting. Morphological and functional data related to PV were collected. The study was approved by the ethical Board of the University of Medicine and Pharmacy Victor Babes from Timisoara and written informed consent was obtained from all participants.

Microbiological stool assessment: Stool samples were collected in sterile containers and sent to the laboratory in maximum $2 \mathrm{~h}$ time interval after collection. Microbiological exam aimed to determine whether species were aerobe, anaerobe or microaerophils and to assess the colony forming units (CFU)/gram stool. The aerobe species: E. coli and E. coli variants, Proteus spp, Klebsiella spp, Enterobacter spp, Serratia spp, Morganella Morganii, Citrobacter spp, Pseudomonas spp, Enterococcus spp and Staphylococcus aureus, Salmonella, Shigella and Yersinia spp. as well as anaerobe species such as Bacteroides spp., Bifidobacterium spp., Lactobacillus spp., Clostridium spp., Clostridium difficile were checked out in all the patients. Cultures for fungi Candida albicans and nonalbicans, Geotrichum spp. were also performed. Species were identified by matrix-assisted laser desorption ionization with MALDI-TOF-MS method[14]. Stool microbiological charts displayed increase or reduction of diverse microorganisms ( + minor, ++ mild and +++ severe), as well as borderline situations $( \pm)$ and normobiosis (physiological condition). GMI was scored: minor (1 point), mild ( 2 points) and severe ( 3 points). Diagnostic and assessment of liver disease severity: Fibromax (Biopredictive) or FibroScan (Echosens) were used to confirm end stage chronic liver disease, with high stage fibrosis ( F4). The assessment of liver cirrhosis severity was based on Child-Pugh score, which employs five variables, two clinical ( ascites and encephalopathy) and three biological (serum albumin, total bilirubin and INR).
Each measure is scored 1-3, with 3 indicating most severe aspects[15,16].

Ascites was graded on a scale of 0 (absence) to 3 (massive) according to amount of liquid : 1-mild , $<600$ CC, 2-moderate 600-1500cc and 3-severe (massive) $>1500-2000 \mathrm{cc}$ [17]. Esophageal varices were graded on a scale of 0 (absence) to 3 (large) according to Paquet classification[18]. Upper digestive bleeding: was scored based on the Forrest classification as absent( 0$)$, minor(1 point $=$ Forest III) , mild ( 2 points $=$ Forest II) and severe $(3$ points $=$ Forrest $\mathrm{I})$, being also classified as variceal or non variceal[19]. Diagnostic of HCC was made according to EASL guidelines, noninvasive criteria were applied in nodules developed in patients with liver cirrhosis[20,21]. Diagnostic of encephalopathy was assessed according to West Haven criteria, based on clinical evaluation, with a score ranging from 0 ( no encephalopathy) to 4 ( severecoma)[22]. Imaging PVT Imagistic studies (abdominal ultrasound, CT/MRI exams) gave details upon thrombus localization, thrombus status (acute/chronic with or withoutcavernous transformation), thrombus obstruction (partial or complete), and its probable etiology (benign or malignant)[ 23]. Severity of PVT was scored as 1 confined to main portal trunk, 2, extension to portal branch 3 extension to splenic or mesenteric veins \pm collaterals development[24]. Abdominal pain was scored as absent (0), mild(1), moderate(2) and severe(3).Short term inpatients outcome ranged from 1 to 4(1: good outcome with status improvement, 2: steady state, 3 : bad outcome, including surgical interventions, 4: death).Statistical Analysis Graph Pad Prism 8 software (Graph Pad Software, Inc., La Jolla, CA, USA) was used to perform statistical analysis. Being an exploratory study no formal sample size calculations were performed, Quantitative variables were expressed as mean values (MV) \pm standard deviation (SD), whereas qualitative variables were expressed as percentages. Chi-squared test was used to compare the two of groups, in cases of categorical analyzed variables. The unpaired ttest was calculated for statistical distribution and interpretation of $\mathrm{p}$ values with confidence interval $\mathrm{Cl}=$ $95 \%, p \leq 0.05$ being considered statistically significant.

\section{Results and discussions}

This observational exploratory pilotstudy was performed on 50 consecutive cirrhotic patients diagnosed with PVT during hospitalization, divided into two groups, after stool exams, as study group (GMI+ patients) and comparison group (GMI -patients). Baseline demographic and biologic aspects: CBC, ALT, ESR,IL-10, creatinin (creat), fasting blood sugar( glic),INR, APTT, D-dimers, albumin (alb),total bilirubin (TB) and GMI are summarized in table 1.

\begin{tabular}{|c|c|c|c|}
\hline & GMI $+(n=25)$ & GMI- $(n=25)$ & $\mathbf{p}$ \\
\hline Age(years) & $70.8 \pm 6.28$ & $52.52 \pm 4.01$ & $=0.0001$ \\
\hline Gender ratio M/W & $76 \% / 24 \%$ & $64 \% / 36 \%$ & $0.3594^{--}$ \\
\hline $\mathrm{U} / \mathrm{R}$ residence & $68 \% / 32 \%$ & $68 \% / 32 \%$ & $1^{-\cdots}$ \\
\hline $\mathrm{Hb}(\mathrm{g} / \mathrm{dl})$ & $10.44 \pm 1.988$ & $11.38 \pm 2.641^{-}$ & 0.1615 \\
\hline $\mathrm{L} / \mathrm{mm}^{3}$ & $6.8 \times 10^{3} \pm 2.746 \times 10^{3-1}$ & $7.08 \times 10^{3}=3.296 \times 10^{3-}$ & 0.7558 \\
\hline $\mathrm{Plt} / \mathrm{mm}^{3-1}$ & $173.08 \times 10^{3}=97.14 \times 10^{3}$ & $218.16 \times 10^{3}=192.34 \times 10^{3}$ & 0.3008 \\
\hline $\operatorname{ALT}(\mathrm{IU})$ & $68.88=66.89$ & $52.84=105.43$ & 0.5289 \\
\hline $\operatorname{ESR}(\mathrm{mm} / \mathrm{h})$ & $45.84=15.02$ & $42.08=19.82$ & 0.4571 \\
\hline IL-10(pg/ml) & $11.488 \pm 2.076$ & $9.680=2.401^{-}$ & 0.0065 \\
\hline Creat(mg/dl) & $1.282 \pm 1.02$ & $1.032 \pm 0.431$ & 0.2652 \\
\hline Glic(mg/dl) & $126.08 \pm 28.65$ & $96.48 \pm 18.77^{-}$ & 0.1706 \\
\hline INR (IÜ) & $1.81=1.08$ & $1.53 \pm 0.6$ & 0.3572 \\
\hline APTT(sec) & $30.24 \pm 9.837$ & $34.42 \pm 17.142$ & $0.2965^{-}$ \\
\hline D-dimers $(\mu \mathrm{g} / \mathrm{ml})$ & $0.5528 \pm 0.2404$ & $0.5432=0.2886$ & 0.8988 \\
\hline $\mathrm{Alb}(\mathrm{g} / \mathrm{l}$ & $3.10 \pm 0.366$ & $3.53 \pm 0.629$ & 0.04 \\
\hline $\mathrm{TB}(\mathrm{mg} / \mathrm{dl})$ & $2.28=2.238$ & $2.17 \pm 3.242$ & 0.8875 \\
\hline
\end{tabular}

\section{Table 1}

DEMOGRAPHIC AND BIOLOGIC CHARACTERISTICS OF RESEARCH PARTICIPANTS Legend: $M / W=$ Men/Women, $U / R=U$ rban/R, $\mathrm{Hb}=$ hemoglobin, $\mathrm{L}=$ leukocytes, PIt= platelets $\mathrm{g} /$ $\mathrm{dL}=$ grams/deciliter, $\mathrm{mm}=$ millimeter, $\mathrm{IU}=$ international units, $\mathrm{mm} / \mathrm{h}=$ millimeters/hour. $\mathrm{pg} / \mathrm{mL}=$ picograms /milliliter, $\mathrm{mg} / \mathrm{dL}=$ milligrams $/$ deciliter, sec=seconds, $\mu \mathrm{g} / \mathrm{mL}=$ micrograms/ milliliter, $p$ bold=statistically significant 
As seen in table I, GMI + patients displayed statistically significant differences only concerning age $(p<0.0001)$, IL- 10 levels $(p=0.0065)$ and lower albumin plasmatic levels $(p=0.04)$. The most suggestive clinical data related to cigarettes smoking and alcohol drinking history, admission, incidentally diagnostic, signs and symptoms, etiology of liver disease, Duplex PV hemodynamic measurements, complications, comorbidities, treatment, as well as outcome and length of hospitalization are illustrated in table 2.

Study group (GMI+) displayed significant differences related to digestive bleeding, abdominal pain severity and longer hospitalization length. The rest of major clinical aspects e.g. smoking and alcohol drinking history, fever, ascites, esophageal varices and variceal bleeding, encephalopathy, severity and etiology of liver cirrhosis, HCC, PV hemodynamic, gastro-intestinal conditions, surgical complications, cardio-vascular comorbidities as well as short term outcome and subsequent in hospital death, showed no significant differences between the two groups. Significant differences concerning clinical short-term outcome were noted in GMI- group, that displayed better clinical short term outcome, with higher percentage of improvement of their condition, when compared to those $\mathrm{GMI}+$, as illustrated in table below (table 3).

A lot of recent studies have found a relation between GMI and thrombosis, being focused mainly on the arterial burden of this condition. Trimethylamine N-oxide (TMAO), a gut microbial metabolite, was linked to enhancement of platelet reactivity and thrombus formation in humans[2527]. However, less is reported regarding the relation between GMI, its subsequent metabolites and possible pathways to venous thrombosis. Small intestinal bacterial overgrow th was associated in a recent retrospective study, as an independent variable, to deep vein thrombosis, diagnosed by duplex examination of lower limbs[28]. The observations of present study showed a strong positive correlation between GMI and PVT severity, hypothesizing that gut dysbioisis could associate a risk for venous thrombosis in portal vein system, as well. Two demographic footprints were identified in patients with PVT: male gender and urban locations. Reliable epidemiological data are still lacking, patients of various ages, including children could develop PVT. The incidence of this condition seems however, to increase in elderly people, male gender being a risk factor for PVT especially in patients with liver cirrhosis[29]. In accordance with others, we often observed an insidious onset, with silent presentation and mostly discrete symptoms[30]. As a consequence, the diagnosis was incidentally made, in more than half of both groups of patients. As reported by previous studies, clinical symptoms at presentation could be variable, depending on many factors, one of the most important being whether the PVT was occlusive or non occlusive[31]. In this series, clinical manifestations were obscure, patients being rarely admitted as emergencies ( $32 \%$ vs. $36 \% ; p=0.7676$ ). Digestive bleeding, as a presentation complaint in GMI+ patients, was seen in a significanthigher number of cases: $48 \%$ vs. $20 \%$ in comparison group of patients $(p=0.03)$. Variceal bleeding in GMI+ was in most of cases of mild importance, consecutively management of digestive bleeding being successful by noninvasively, endoscopic approach. Some authors reported more severe digestive bleeding in series of patients with extrahepatic and occlusive PVT, requiring various hemostasis procedures[32]. Liver cirrhosis is a known risk factor for

\begin{tabular}{|c|c|c|c|}
\hline & GMI + (n=25) & GMI- $(n=25)$ & p \\
\hline Smoking history & $60 \%$ & $40 \%$ & 0.1615 \\
\hline Alcohol drinking history & $56 \%$ & $52 \%$ & 0.7788 \\
\hline Admission as emergency & $32 \%$ & $36 \%$ & 0.7676 \\
\hline Incidentally diagnostic & $56 \%$ & $52 \%$ & 0.7788 \\
\hline Digestive bleeding presence & $48 \%$ & $20 \%$ & 0.03 \\
\hline Digestive bleeding severity & $0.84 \pm 0.69$ & $0.56 \pm 0.65$ & 0.1458 \\
\hline Variceal bleeding & $20 \%$ & $12 \%$ & 0.4450 \\
\hline Esophageal varices & $0.92 \pm 0.86$ & $0.80=0.71$ & 0.5930 \\
\hline Abdominal pain severity & $1.96 \pm 0.79$ & $1.12=0.93$ & $0.001^{-1}$ \\
\hline Fever & $44 \%$ & $36 \%$ & 0.5676 \\
\hline Ascites & $1.56 \pm 0.92$ & $1.36 \pm 1.22$ & 0.5155 \\
\hline Encephalopathy grading & $0.64 \pm 0.95$ & $0.4 \pm 0.87$ & 0.3558 \\
\hline Child $A$ & $24 \%$ & $20 \%$ & 0.9573 \\
\hline Child B & $32 \%$ & $36 \%$ & 0.8444 \\
\hline Child C & $44 \%$ & $44 \%$ & 1 \\
\hline Viral etiology & $20 \%$ & $24 \%$ & 0.7354 \\
\hline Toxic etiology (alcohol) & $44 \%$ & $48 \%$ & 0.7788 \\
\hline Mixed (toxic/viral) etiology & $12 \%$ & 20 & 0.4450 \\
\hline Miscellaneous (NASH) & 24 & $8 \%$ & 0.1266 \\
\hline $\mathrm{PV} \mathbf{v}(\mathrm{cm} / \mathrm{sec})$ & $18.24=5.37$ & $20.56 \pm 6.05$ & 0.1581 \\
\hline Primary liver cancer (HCC) & $52 \%$ & $32 \%$ & 0.1561 \\
\hline Associated hematological conditions & $16 \%$ & $8 \%$ & 0.3889 \\
\hline Associated g-i conditions & $36 \%$ & $20 \%$ & 0.2123 \\
\hline $\mathrm{ACT}$ & $40 \%$ & $44 \%$ & 0.7767 \\
\hline Inpatients mortality & $8 \%$ & $8 \%$ & 1 \\
\hline Transfer to surgery & $12 \%$ & $8 \%$ & 0.3020 \\
\hline $\mathrm{C}-\mathrm{V}$ comorbidities & $44 \%$ & $36 \%$ & 0.5676 \\
\hline Outcome scoring & $2.12 \pm 0.73$ & $2=0.91$ & 0.6093 \\
\hline PVT severity & $2.20=0.65$ & $2.12=0.60$ & 0.6520 \\
\hline Length of hospitalization (days) & $8.16 \pm 4.22$ & $5.80=2.93$ & 0.02 \\
\hline
\end{tabular}

\section{Table 2}

CLINICAL PARTICULARITIES IN CIRRHOTIC PATIENTS WITH PVT

Legend: $\mathrm{NASH}=$ nonalcoholic steatohepatitis, PVv $(\mathrm{cm} / \mathrm{sec})=$ portal vein velocity (centimeters/ second), $\mathrm{HCC}=$ hepatocellular carcinoma, $g-i$ = gastrointestinal, $\mathrm{ACT}=$ anticoagulant treatment, $\mathrm{c}-\mathrm{v}=$ cardio-vascular, $\mathrm{p}$ bold $=$ statistically significant

\begin{tabular}{|c|c|c|c|}
\hline Outcome & GMI+ (n=25) & GMI- (n=25) & p \\
\hline 1 & $12 \%$ & $40 \%$ & $\mathbf{0 . 0 2 5 5}$ \\
\hline 2 & $68 \%$ & $44 \%$ & 0.09 \\
\hline 3 & $12 \%$ & $8 \%$ & $0.6407^{-}$ \\
\hline 4 & $8 \%$ & $8 \%$ & 1 \\
\hline
\end{tabular}

Table 3

SHORT TERM CLINICAL OUTCOME IN RESEARCH PARTICIPANTS

Legend: Outcome $1=$ improvement, $2=$ steady state, $3=$ aggravation, $4=$ death, $p$ bold=statistically significant 
PVT, the association of PVT to end stage chronic liver diseases being reported in $25-40 \%$ of cases[33]. In a J apanese retrospective study, patients with liver cirrhosis due to viral hepatitis experienced PVT in $40 \%$ of cases over 10 years follow-up[34]. High levels of circulating lipoproteins (LPSS) were recently reported in liver cirrhosis patients with PVT[35]. Our observations group bring up to discussion that not only bacterial translocation generating LPSs, but also gut microbiota dysbiosis and its own metabolic consequences could be involved in thrombogenic process. The vast majority of patients presented with chronic, non occlusive forms of PVT that could explain a relatively good short-term outcome, especially for GMI- patients, but no strong correlations between PVT, GMI and short term outcome were noted in study group population.As other studies reported, even before liver transplantation, patients with advanced liver diseases and PVT may not necessarily have a worse outcome[36,37]. Recent studies related to cardio-vascular risk events demonstrated that some DB gut signature seemed to be associated to a particular phenotype microbial imbalance, that results in increase of circulating LPSs with emerging pro-inflammatory and prothrombogenic responses. Through generation of TMAO, gut dysbiosis could contribute to enhancement of platelets reactivity and increases the risk for thrombosis.[38] Our observations related to GMI, IL-10 and D-dimers level in our study population ( $\mathrm{GMI}+$ ) emphasizing that from clinical point of view elevation of $D$-dimers could express a particular gut dysbiotic signature with enhancement of inflammatory and thrombogenic process that could mandate some therapeutic approaches accordingly. Is it possible to alleviate the burden of GMI and its consequences, including the thrombogenic risk? A very appealing idea could be overcoming GMI burden by targeting gut microbiota metabolites. Targeting TMAO whether by dietary changes or by pre and probiotics administration, or orally low absorbable antibiotics, could influence platelets behavior, resulting in mitigation of aggregation, along with decreasing the thrombogenic risk, without inducing alterations of platelets function. In this scenario patients will not be at risk for bleeding, when compare to those receiving antiaggregant or anticoagulant treatment.[39,40] Further approach should address this topic in prospective studies.

This observational pilot study explored a potential exposure to GMI, in patients with liver cirrhosis diagnosed with PVT during hospitalization, providing only preliminary data regarding some clinical particularities that could result in burden increase. Further approaches should address this topic in prospective studies.

In conclusion, cirrhotic patients with GMI displayed clinical particularities characterized by older age, higher inflammatorystatus, hypoalbuminemia, more severe PVT, abdominal pain and digestive bleeding, as well as longer hospitalization duration.

\section{References}

1.A KUMAR, P SHARMA, A Arora Portal vein obstruction- Epidemiology, Pathogenesis, Natural History, Prognosis and Treatment Aliment Pharmacol Ther, 2/11/15

2.WOLBERG AS, ALERMAN M, LEIDERMAN K, MACHLUS KL Procoagulant activity in hemostasis and thrombosis: Virchow 's triad revisited.Anesth Analg. 2012:114(2):275-285.

3.SIRLI R, SPOREA I, POPESCU A, DANILA M. Ultrasound-based elastography for the diagnosis of portal hypertension in cirrhotics World J Gastroenterol 2015;21(41):11542-51
4.LESSIER A, DARWISH-MURAT S, HERNANDES GUERRA $N$ et al. European Network for Vascular Disorders of the Liver (EN-Vie)Acute portal vein thrombosis unrelated to cirrhosis, a prospective multicenter follow-up study. Hepatology 2010;51-210-218

5.CHAWLA YK BODH V Portal vein thrombosis J Clin Exp Hepatol 2015;5:22-40

6.VALLA DC, CONDAT B. Portal vein thrombosis in adults: pathophysiology, pathogenesis and management. J Hepatol. 2000;32:865-871.

7.ZOLI M, IERVESE T, MERCKEL C, et al. Prognostic significance of portal hemodynamic in patients with compensated cirrhosis. J of Hepatol 1993; 17:61.

8.LEE EYP, KHONG PL The value of 18 FDG PET scan in detection of tumor thrombus .Clin Nucl Med 2013; 38:e60-5.

9.DE FRANCHIS R BAVERNO V FACULTY, Revising consensus workshop on methodology of diagnosis and therapy in portal hypertension. J of Hepatol 2010;53: 762-8.

10.PONZIANI FR, ZOCCO MA, CAMPANELE C, et al. Portal vein thrombosis: insight into physiopathology and treatment. World J Gastroenterol 2010; 16: 143-55.

11.OGREN M, BERGOVITS D, BYORCK M, ACOSTA S et al. Portal vein thrombosis, patients characteristics and life time risk: a population study based on 23796 consecutive autopsies. World J Gastroenterol.2006;12:2115-2119.

12.CONDAT B, PESSIONE F, HILLAIRE S, DENNINGER MH, GUILLIN MC, POLIQUIN M, HADENGUE A, ERLINGER S, VALLA D. Current outcome of portal vein thrombosis in adults: risk and benefit of anticoagulant therapy. Gastroenterology. 2001;120:490-497.

13.MARTINEZ-DEL CAMPO A, ROMANO A K, REY EF, BALSKUS EP The Plot Thickens: Diet Microbe Interactions May Modulate Thrombosis Risk Cell Metabolism, 2016; 23(4): 573-575

14.SANDRIN TR, GOLDSTEIN JE AND SHOEMAKER S. MALDI TOF MS profiling of bacteria at the strain level: A review. Mass Spectrometry Reviews 2013;32(3):188-217

15. CHILD CG, TURCOTTE J G Surgery and portal hypertension. In Child CG. The liver and portal hypertension. Philadelphia: Saunders. 1964);pp. 50-64

16.PUGH RN, MURRAY-LYON IM, DAWSON JL, PIETRONI MC, WILLIAMS R Transection of the oesophagus for bleeding oesophageal varices British J of surgery. 1973; 60 (8): 646-9

17.ENNIS J, SCHULTZG, PERERA P, WILLIAMS S et al Ultrasound for Detection of Ascites and for Guidance of the Paracentesis Procedure: Technique and Review of the Literature. Internat J Cl Med 2014; 5: 1277-1293

18.PAQUET, KJ . Prophylactic endoscopic sclerosing treatment of the esophageal wall in varices - A Prospective Controlled Randomized Trial. Endoscopy 1982; 14: 4-5

19. FORREST JA, FINLAYSON ND, SHEARMAN DJ. Endoscopy in gastrointestinal bleeding. Lancet. 1974;2:394-397

20.SERSTE T, BARRAU V, OZENNE $V$ et al Accuracy and disagreement of computed tomography and magnetic resonance imaging for the diagnosis of small hepatocellular carcinoma and dysplastic nodules: role of biopsy. Hepatology 2012;55:800-806

21.EUROPEAN ASSOCIATION FOR THE STUDY OF THE LIVER; European Organisation for Research and Treatment of Cancer. EASL-EORTC clinical practice guidelines: management of hepatocellular carcinoma. J Hepatol 2012;56:908-943

22. FERENCI P, LOCKWOOD A, MULLEN $K$ et al Hepatic encephalopathy-definition, nomenclature, diagnosis, and quantification: final report of the working party at the 11th World Congresses of Gastroenterology, Vienna, 1998. Hepatology. 2002;35:71621

23.GEORGESCU D, GEORGESCU LA, MUNTEAN M, LIGHEZAN D. Duplex abdominal examination in portal vein obstruction: how much can we rely on? Ultraschall in der Medizin 2016;37:(S01)SL13_5.

24. LEE HK, PARK SJ , YI BH, et al. Portal vein thrombosis: CT features. Abdom Imaging. 2008;33(1):72-79 
25.ZHU W, et al Gut Microbial Metabolite TMAO Enhances Platelet Hyperreactivity and Thrombosis Risk. Cell. 2016;165(1):111-124. 26.ZHU W, WANG Z, TANG WHW, HAZEN SL. Gut Microbe-Generated Trimethylamine N-Oxide From Dietary Choline Is Prothrombotic in Subjects. Circulation. 2017;135(17):1671-1673

27.SENTHONG V, LI XS, HUDEC T et al. Plasma TMAO, a gut microbe generated metabolite, is associated with atherosclerotic burden. J Am Coll Cardiol 2016; 67:2620-2628

28.FIALHO A, FIALHO A, SCHENONE A et al. Association between small intestinal bacterial overgrowth and deep vein thrombosis. Gastroenterol Rep 2016;4(4):299-303

29.AMITRANO L, GUARDASCIONE MA, BRANCACCIO V et al. Risk factors and clinical presentation of portal vein thrombosis in patients with liver cirrhosis. J Hepatol.2004; 40:736-741.

30.WEBSTER GJ, BURROUGHS AK, RIORDAN SM, Portal vein thrombosis: new insight into etiology and management.Aliment Pharmacol Ther. 2005;21:1-9.

31.QI X,SU C, REN W et al Association between portal vein thrombosis and risk of bleeding in liver cirrhosis: a systematic review of the literature Clin Res Hepatol Gastroenterol 2015;39:683-691

32.J ANSSEN HL, WIJHOUD A, HAASMA EB et al. Extrahepatic portal vein thrombosis: etiology and determinants of survival. Gut 2001;48:720-724.

33.PLESSIER A, DARWISH-MURAD S, HERNANDEZ GUERRA M et al. European Network for Vascular Disorders of the Liver(EN-Vie).Acute portal vein thrombosis unrelated to cirrhosis: a prospective multicenter follow-up study. Hepatology 2010;51:210-218]
34.MARUYAMA H, OKUGAWA H, TAKAHSHI M etal De novo portal vein thrombosis in virus related cirrhosis: predictive factors and long term outcomes. Am J Gatroenterol 2013;108:568-574

35.MALAGUARNERA G, CATANIA VE, FRANCAVIGLIA A et al. Lipoprotein(a) in patients with hepatocellular carcinoma and portal vein thrombosis Aging Clin Experim Res 2017; 29( Suppl 1):185190

36.DENNINGER MH,CHAIT Y, CASADEVAL $N$ et al. Cause of portal or hepatic vein thrombosis in adults: the role of multiple concurrent factors. Hepatology.2000; 31:587-591.

37. CHEN H, TURON F, HERNÁNDEZGEA V, FUSTERJ, GARCIA CRIADO A, BARRUFET $M$, DARNELL $A$ et al. Nontumoral portal vein thrombosis in patients awaiting liver transplantation Liver Transpl 2016;22(3):352-365

38. FALONY G, VIEIRA-SILVA S, RAES J Microbiology Meets Big Data: The Case of Gut Microbiota-Derived Trimethylamine. Annu Rev Microbiol. 2015;69:305-21

39.ROBERTS AB, GU X, BUFFA JA et al. Development of a gut microbetargeted nonlethal therapeutic to inhibit thrombosis potential Nat Med 2018;24:1407-1417

40.STIKSRUD B, NOWAK P, NWOSU F C. KVALE D, THALME A, SONNERBORG A et al. Reduced Levels of D-dimer and Changes in Gut Microbiota Composition After Probiotic Intervention in HIVInfected Individuals on Stable ART J Acquir Immune Defic Syndr 2015;70(4):329-337

Manuscript received: 28.12 .2018 